Revista Arbitrada Interdisciplinaria KOINONIA

Año 2020 . Vol V. $\mathrm{N}^{\circ} 1$. Especial Educación

Hecho el depósito de Ley: FA2016000010

ISSN: 2542-3088

FUNDACIÓN KOINONIA (F.K). Santa Ana de Coro. Venezuela.

Mirian-Del-Rocío González Espinoza; Darwin Gabriel García-Herrera; Cristián Andrés Erazo-Álvarez; Juan Carlos Erazo-Álvarez

http://dx.doi.org/10.35381/r.k.v5i1.797

\title{
Creatividad y técnicas grafoplástica innovadoras
}

Creativity and innovative graphoplasty techniques

\author{
Mirian-Del-Rocío González Espinoza \\ mirian.gonzalez@psg.ucacue.edu.ec \\ Universidad Católica de Cuenca, Azogues \\ Ecuador \\ https://orcid.org/0000-0001-5082-8437 \\ Darwin Gabriel García-Herrera \\ dggarciah@ucacue.edu.ec \\ Universidad Católica de Cuenca, Azogues \\ Ecuador \\ https://orcid.org/0000-0001-6813-8100 \\ Cristián Andrés Erazo-Álvarez \\ cristianerazo@ucacue.edu.ec \\ Universidad Católica de Cuenca, Cuenca \\ Ecuador \\ https://orcid.org/0000-0001-8746-4788 \\ Juan Carlos Erazo-Álvarez \\ jcerazo@ucacue.edu.ec \\ Universidad Católica de Cuenca, Cuenca \\ Ecuador \\ https://orcid.org/0000-0001-6480-2270
}

Recibido: 26 de abril de 2020

Revisado: 25 de mayo de 2020

Aprobado: 15 de junio de 2020

Publicado: 30 de junio de 2020 


\title{
RESUMEN
}

Partiendo del hecho de que las técnicas grafo plásticas son fundamentales para el proceso de enseñanza-aprendizaje de los niños y niñas en el nivel inicial de educación, el presente trabajo investigativo se plantea con el objetivo de identificar cuáles son las estrategias que utilizan las educadoras de los centros de desarrollo infantil, para desarrollar la creatividad en los niños y niñas de 12 a 36 meses, mediante la aplicación de una investigación no experimental, descriptiva con enfoque mixto y de cohorte transversal, para con base en los resultados plantear alternativas, que mejoren los conocimientos y destrezas de las educadoras en cuanto a la aplicación de técnicas grafoplásticas innovadoras en base a una metodología didáctica, que permitan desarrollar la creatividad implícita en cada ser humano y más en edades tempranas.

Descriptores: Artes gráficas; educación artística; educación de la primera infancia; docente de preescolar. (Palabras tomadas del Tesauro UNESCO).

\begin{abstract}
Starting from the fact that graphical plastic techniques are fundamental for the teachinglearning process of boys and girls at the initial level of education, this research work is proposed with the aim of identifying which are the strategies that educators use child development centers, to develop creativity in boys and girls aged 12 to 36 months, through the application of non-experimental, descriptive research with a mixed approach and a cross-sectional cohort, based on the results, proposing alternatives that improve the knowledge and skills of the educators regarding the application of innovative graphoplastic techniques based on a didactic methodology, which allow developing the creativity implicit in each human being and more at early ages.
\end{abstract}

Descriptors: Graphic arts; art education; early childhood education; preschool teachers. (Words taken from the UNESCO Thesaurus).

\section{INTRODUCCIÓN}

Las técnicas grafoplásticas nacieron con el fin de que los niños puedan desarrollar la creatividad artística mediante la motricidad fina, debido a que cuando se aplican las técnicas desde edades muy tempranas los niños han demostrado notablemente que la creatividad artística se ha aumentado positivamente debido a que se realiza la oportuna 
motivación por medio del desempeño de forma manual el niño podrá visualizar un nuevo rumbo de educación que es guiado principalmente en el desarrollo de técnicas grafoplásticas basado en la creatividad artística (Gancino, 2012).

Es interesante mencionar que en Ecuador por medio de la (Asamblena Nacional Constituyente, 2012) y el Ministerio de Educación adquieren la aplicación del sistema educativo RETEC desde el año de 1999, proyecto español que permite desarrollar las habilidades artísticas dirigido a los niños adjuntos al sistema educativo a nivel nacional, pero lamentablemente la aplicación de las técnicas grafoplásticas no han sido consideradas como importantes, en los establecimientos educativos que dependen del Estado Ecuatoriano (Ministerio de Educación del Ecuador, 2019).

El Nuevo Plan Decenal de Educación (Educiudadania, 2016) es el instrumento estratégico de gestión y una guía que da perspectiva a la educación, una de ellas es la Política 3, que dice: Universalización de la Educación Inicial, para dotar a los infantes de habilidades para el acceso y permanencia en la educación básica (Subsecretaría de Educación Inclusiva, 2019). Es considerable pensar que los primeros años de vida de un ser humano, recibe estimulación: afectiva, cognitiva y motriz. Hecho que una serie de investigaciones y experimentaciones científicas revelan la necesidad de atender las necesidades de cada uno de los infantes y su desarrollo de las áreas psicomotrices.

Ante lo expuesto, la visión tanto de docentes como parvularias que están a cargo de niños y niñas en nivel pre escolar, que tienen respecto de las artes plásticas como un período de aprestamiento para el proceso de la lectura y escritura, factor principal para el desarrollo de la motricidad fina, más no las consideran como el nexo o puente directo para lograr el desarrollo de la motivación y creatividad en los niños y niñas y en la ciudad de Azogues la mayoría de las instituciones educativas no le dan la importancia debida al primer año de Educación General Básica, limitando al niño a la realización de actividades lúdicas que desarrollen su creatividad.

Es considerable pensar la importancia de la motricidad en la educación infantil que tiende a favorecer el dominio del movimiento corporal, la comunicación que se establece con el mundo que le rodea, la interacción personal, las relaciones témporo espaciales 
fundamentales en el desarrollo de la movilidad, motivación y creatividad de los niños y niñas.

En Azogues, existe solamente una escuela del milenio creada recientemente donde se encuentra los espacios adecuados para que las técnicas grafoplásticas puedan ser aplicadas para desarrollar las habilidades artísticas importantes que dirigen el desarrollo de la creatividad e imaginación de los niños, fomentando individuos críticos, seguros y creativos, por lo que conocer su problemática nos ayudará a identificar y tratar oportunamente determinadas situaciones, para dar alternativas de solución ante las mismas, por lo tanto, nos permite: entenderlos, valorarlos e integrarlos en el nivel que corresponde dentro de los procesos de enseñanza aprendizaje (Ministerio de Educación del Ecuador, 2019), mejorando el desarrollo de la creatividad en los niños y niñas pero no se enfoca en los infantes de 12 a 36 meses, por ello la necesidad de que el docente debe convertirse en guía del aprendizaje significativo del niño aplicando técnicas constructivistas para que los niños puedan pensar y diseñar sus conocimientos.

Entonces, los primeros años de vida del infante en formación es la base fundamental debido a que es el instante en que el niño comienza a experimentar y desarrollar habilidades y destrezas, así como su imaginación y creatividad lo que permitirá crecer hacia una vida estudiantil exitosa y responsable; por ende, se hace necesario contar con profesionales capacitados no solo con el conocimiento técnico sino también con actitudes y aptitudes acordes a las exigencias actuales que son exigidas por el gobierno, sociedad y particularmente por las instituciones educativas, al formar infantes que puedan potenciar el proceso de enseñanza-aprendizaje.

Por cuanto los infantes al desarrollar ciertos procesos mentales como: explorar, buscar, manipular, diferenciar, comparar y experimentar estarán listos para crear y sobretodo resolver determinadas situaciones de aprendizaje y activamente poder compartir socialmente con todos los que los rodean en cualquier ambiente que se encuentren; de ahí el hecho de la importancia de desarrollar la creatividad a través de las técnicas grafoplásticas innovadoras en los niños que acuden a los centros de desarrollo infantil en edades entre 12 a 36 meses. 
Asimismo, la creatividad es la forma más sencilla y directa para que el infante pueda expresar y desarrollar las habilidades propias de la motricidad fina considerando también el hecho de que la educación está tomando un giro, al pretender que los alumnos dejen de ser una especie de robot que repiten e imitan a los adultos sino más bien a que sean creativos, reflexivos, analíticos, participativos y porque no inventores mejorando así la calidad educativa.

Además, el interés para potenciar la práctica docente mediante una didáctica adecuada permitirá formar la enseñanza basada en una Pedagogía activa para que los docentes tengan los recursos necesarios idóneos mejorando la calidad de la educación; y, a su vez, mejorar el trabajo en el aula con los niños por medio de las técnicas grafoplásticas fortaleciendo sus capacidades como la imaginación, la creatividad, motricidad, empatía entre otros durante el proceso de aprendizaje.

Además, es importante que el personal docente especialmente de los centros infantiles: Dr. César Molina del MIES y CEI Particular Universitario de Azogues, estén siempre en constante superación al optimizar el desempeño laboral por medio del mejoramiento académico y la respectiva auto capacitación acerca de la inserción activa de las técnicas grafoplásticas garantizando un proceso de enseñanza-aprendizaje eficaz que promueven que los niños y niñas sean creativos, críticos, con autoestima positiva que poco a poco van demostrando el impulso de intentar cosas nuevas, experimentar nuevas actividades, colaborar con los demás, reconocer sus errores y aprender de ellos; por tal motivo los infantes que acuden al centro de Desarrollo Infantil Dr. César Molina y CEI Particular Universitario Católico, son los que se benefician creando nuevas habilidades que incentivan el desarrollo de la creatividad artística.

Asimismo, es de suma importancia describir un breve concepto de las Técnicas GrafoPlásticas (Hussey, 2017), las técnicas grafo plásticas son las "estrategias que se utilizan en los primeros años de educación básica para desarrollar la psicomotricidad fina en los infantes para el desarrollo afectivo e intelectual, los docentes requieren de estrategias y métodos para llegar con los aprendizajes a los niños". 
Desde lo planteado, el objetivo de la investigación es conocer que estrategias utilizan las educadoras de los Centros Infantiles relacionadas con las técnicas grafoplásticas para desarrollo de la creatividad en los niños y niñas de 12 a 36 meses, específicamente en los centros Dr. César Molina y 11 del CEI Particular Universitario de Azogues.

\section{Referencial teórico}

\section{Fundamentos teóricos}

En el Ecuador, desde el año 2013 el Ministerio de Educación se ha empeñado integrar a los infantes, desde tempranas edades a la educación formal; dando importancia al arte, por lo que, la expresión plástica y artística debería ser manejada debidamente por los docentes para buscar no solo la potencialización de las áreas de motricidad fina y gruesa sino para la expresión de la creatividad (Ministerio de Educación del Ecuador, 2019).

\section{El desarrollo de la estimulación temprana}

Consite en hacer uso de todos los recursos para emplear en los infantes técnicas que conlleve a que se desarrolle las capacidades cognitivas fisicas al maximo, evaluando constantemente para evitar posibles retrasos de aprendizaje con lo que se conseguira que los niños puedan, pensar, acutar y sentir todas las experiencias posibles que vayan adquiriendo, por ende, se debe tener muy en claro cuales son las actividades que el niño requiere para potenciar dichas capacidades, además los beneficios que ofrece la estimulación temprana contempla aspecto como lo menciona (Gil Madrona, 2008).

1. Promueve deterinadas condiciones educativas, fisiológicas, recreativas y sociales.

2. La estimulación en el proceso de aprendizaje en las áreas, psicomotriz, afectiva e intelectual.

3. Aumenta la curiosidad por medio de la observación para conocer e interpretar el habitad en el que se encentran.

4. Los lazos afectivos entre los familiares especialemente entre los padres.

5. En ciertos casos, logra identificar posibles trastornos de aprendizaje.

Por lo que, es de vital importancia que la estimulación temprana en los niños que acuden 
a los centros de desarrollo infantil puedan ser evaluados constamente y que por medio de las tecnicas grafoplasticas innovadoras es posible potenciarlizar las habilidades y destrezsas innatas en cada pequeño ser.

\section{Motricidad fina}

El desarrollo de la motricidad fina en los niños y niñas del grado preescolar, permite realizar movimientos pequeños de manos, muñeca y dedos permitiendo al cerebro interpretar emociones y sentimientos, haciendo también referencia a la coordinación de las funciones neurológicas, esqueléticas y musculares utilizadas para producir movimientos precisos (Cabrera \& Garcia, 2019).

Chuva (Chuva Castillo, 2016) en su trabajo de investigación dice que: El niño debe pasar por tres etapas sumamente importantes para desarrollar correctamente a la motricidad fina. Etapa inicial (a través de la observación el niño puede alcanzar un modelo o imitación), Etapa intermedia (el niño mejora la coordinación y el desempeño de los movimientos, Etapa madura (el niño integra todos los elementos de los movimientos en una acción bien ordenada e intencionada).

La educación está avanzando acorde a las exigencias de un mundo globalizado por lo que el enfocarse en la educación inicial es sin lugar a duda una de las etapas de mayor consideración e importancia debido a que es donde se inicia el entrenamiento para el fortalecer en especial la motricidad fina, siendo entonces necesario, que los centros de desarrollo infantil contemplen todas las estrategias posibles que hagan del infante un ser que pueda tener una vida saludable (Gamarra \& Navas, 2017).

\section{Importancia de la motricidad fina}

La estimulación para el desarrollo de la motricidad fina es importante durante los primeros años de vida, que para poder escribir o realizar determinada actividad se requiere una conexión entre la coordinación y el entrenamiento motriz de la mano, siendo de gran importancia que los docentes deben priorizar a llevar a cabo todas las actividades que puedan emprender las habilidades y destrezas requeridas para que los músculos finos tanto de las mano como de los dedos puedan ser desarrollados satisfactoriamente. 


\section{Clasificación de la motricidad fina}

El desarrollo de la motricidad fina en los niños y niñas del grado preescolar (Cabrera \& Garcia, 2019) realiza la siguiente clasificación:

Coordinación viso manual. - Todos los movimientos que fluyen con la vista y la mano, habilidades cognitivas que son guiadas por los diferentes movimientos de la mano acorde a los estímulos percibidos por la vista. Se debe acotar que el infante en sus primeros meses de nacido se les debe ofrecer ciertos materiales para que los niños se sientan cómodos y puedan trabajar a gusto con la creatividad que hayan desarrollado.

Motricidad Facial. - Se referiré a cada uno de los movimientos de los músculos faciales como son los labios, la lengua, la boca con el fin de poder identificar que característica en especial posee el niño y por consiguiente proceder a trabajar en desarrollar y fortalecer la motricidad fina.

Motricidad Fonética. Se refiere al proceso para desarrollar el lenguaje del infante de acuerdo al ritmo del niño tomando en consideración la edad, por lo que se debe buscar las estrategias y técnicas grafoplásticas que lo permitan. Es de vital importancia debido a que en esta etapa los infantes podrán vocalizar correctamente hasta llegar a su etapa de maduración.

Materiales y Técnicas de Expresión Grafo - Plásticas: Entre los materiales más usados tenemos: lápices de grafito, lápices de colores, lápices compuestos, crayones de colores, hojas blancas, témperas, pinceles, hisopos, plastilina, anilina, tizas blancas y de color, pintura de agua, material reciclable, masa, tijeras, gomero, peinillas cepillos, rompecabezas entre otros.

Tipos de Técnicas Grafo Plásticas: Entre las más importantes están: Garabateo, Trozado, Rasgado, Arrugado, Dactilopintura, Modelado, Picado, Plegado, Tijera y Cortado, Rompecabezas, Collage.

\section{Descripción de los Tipos de Técnicas Grafo Plásticas}

Modelado. Técnica innovadora que se inmiscuye en lo tridimensional al incorporar el volumen y la profundidad promoviendo una experiencia nueva al explorar lo sensorial 
utilizando recursos como la masa, arena, plastilina y la macilla; materiales que fortalecen los músculos pertenecientes a la mano y a su vez permite la canalización de sentimientos que pueden ser considerados como agresivos.

Dáctilo pintura. Técnica que ofrece una vía de liberación o escape y sobretodo explota la experimentación sensorial, táctil, visual y kinestésica por medio de pintar utilizando una o todas las partes de la mano y pies, para ello se prepara una mezcla que contenga color donde el infante tiene total libertad de hacer uso tanto de sus manos como de sus pies para explotar no solo sus conocimientos y habilidades sino también aprenden que se pueden ensuciarse libremente y sin temor alguno lo que permite que se desarrolle la expresión e incluso la personalidad infantil.

Grabado. Técnica basada en la impresión para ello se escoge una imagen para ser trasferida la imagen que puede ser dibujada de varias formas de acuerdo al uso de instrumentos y materiales como son manos, solo los dedos, tapas, palillos lo que facilita que el niño pueda identificar las diferencias de las variadas imágenes que se puedan lograr.

Collage. Es una técnica que consiste en pegar trozos de tela, papel, revistas crepe, semillas, fotografía, hojas, cartones, periódicos, cometas y demás objetos que se colocan sobre una superficie plana, donde se van superponiendo consiguiendo construir figuras variadas además que puede combinarse con otras técnicas grafoplásticas para completar el desarrollo físico, motriz y sensorial del niño.

Construcción. - Técnica que ofrece el poder identificar colores, formas y tamaños de los objetos que manipule compuestos de uno o varios materiales, esto permite fortalecer habilidades y destrezas como el lenguaje en conjunto con la motricidad fina y grueso del niño.

Técnicas Didácticas: Son el medio de participación de dinamismo, que consiste en activar el proceso enseñanza aprendizaje, que requieren los infantes para su crecimiento personal, es evidente que una clase donde se ponga en funcionalidad las técnicas permitirá superar las dificultades de asimilación de los contenidos tratados durante el período clase (Murrillo, 2012). 
Desarrollo Educativo: Cada uno de los factores que se involucran en la profesionalización del ser humano, educando, desde el inicio hasta el final del cumplimiento de sus metas y propuestas.

\section{Desarrollo creatividad artística}

"Integración de habilidades adquiridas, para desarrollar la creatividad, buscando la integración de los saberes, equilibrando los desempeños, fortaleciendo la creatividad y la imaginación" (Castañeda, 2013, p. 3).

El desarrollo artístico en el niño permite el fortalecimiento de las destrezas adquiridas y conseguir nuevas habilidades mediante la práctica constante contribuyendo con la formación integral de los conocimientos, desarrollando la creatividad de los infantes para ello es importante que el niño se desenvuelva en un ambiente amigable y de aprendizaje. El desarrollo de la creatividad en los niños desde sus primeros años es importante porque estimula al desarrollo de habilidades, adquiere nuevas destrezas, incrementa el potencial imaginativo donde plasma sus emociones y pensamiento en dibujos y pinturas, integra sus saberes con el arte artístico. Es importante la preparación y capacitación del docente para que contribuya con la formación integral del niño a través de la educación artística que permite aprender de una manera divertida y entretenida debido a que ayuda a mejorar aspectos educativos y de relaciones personales logrando un mejor desenvolvimiento en la sociedad y pueda tomar decisiones correctas en la vida al tener un criterio formado.

Expresión artística: La expresión plástica es uno de los mecanismos favoritos del ser humano para expresar sus ideas. Desde nuestros antepasados se han encontrado escrituras con pequeños dibujos que representaban palabras o incluso frases completas mediante las cuales los pueblos se comunicaban, cuando aún no existía el lenguaje oral. La plástica es una herramienta básica para la comunicación que hoy en día ocupa un lugar privilegiado en los distintos niveles de la educación (Castañeda, 2013).

(Physical Development and Health, 2017), la revista estadounidense señala que los niños que inician la actividad escolar deben ir ganando destrezas como la coordinación de manos, ojos, fuerza y flexibilidad cuando el infante utiliza tanto las manos como los dedos 
para trabajar haciendo uso de diferentes tipos de materiales y objetos, ideas que incentivan la motivación convirtiendo a los infantes en pequeños seres llenos de habilidades que van desarrollando en unos casos y en otros descubriéndolas para luego ser incrementadas asegurando una buena formación física y mental de los pequeños seres que comienzan a experimentar del medio en el que se desenvuelven.

\section{Condiciones del desarrollo de la creatividad}

Externas: Como la seguridad psicológica donde el niño y la niña necesitan de un ambiente en el cual sean aceptados incondicionalmente. Esto significa que el docente debe aceptar a cada uno de sus dicentes tal cual son. (Larrea, 2013) al sentirse el niño y la niña desde un principio aceptados, podrá desarrollar su creatividad.

Condiciones internas: Como la libertad Psicológica: Cuando al niño y a la niña se les brinda la libertad que requiere la expresión simbólica, entonces surge la creatividad, expresar libremente lo que siente. Esta libertad no debe confundirse con permisividad; el docente debe facilitar el ser libres, pero con responsabilidad, de esta manera pueden también existir la confusión, equivocación y temor.

Funciones del desarrollo artístico: Función comunicativa, pues el niño/a, a través de su expresión pretende comunicar vivencias, experiencias y sus sentimientos, función de representación, la que ocurre en la mente del niño y lo interpreta y la representación plástica, plasmará sus emociones, y función lúdico-creativa: explora un material nuevo con el que tienen la posibilidad de hacer nuevas creaciones y disfrutar jugando (Larrea, 2013).

\section{Para Evaluar la creatividad}

Se deben tener en consideración criterios e indicadores que utilizan las educadoras para desarrollar la creatividad como originalidad, mediante la cual se fomenta la capacidad del niño a ser creativo e innovador. La iniciativa es otro indicador a tener en consideración, por cuanto es el factor que permite generar las ideas que sustentan los emprendimientos, siendo participe de la fluidez como proceso que determina la cantidad y calidad de expresiones espontaneas por parte del niño, siendo necesaria evaluar la flexibilidad como 
Revista Arbitrada Interdisciplinaria KOINONIA

Año 2020 . Vol V. $\mathrm{N}^{\circ} 1$. Especial Educación

Hecho el depósito de Ley: FA2016000010

ISSN: 2542-3088

FUNDACIÓN KOINONIA (F.K). Santa Ana de Coro. Venezuela.

Mirian-Del-Rocío González Espinoza; Darwin Gabriel García-Herrera; Cristián Andrés Erazo-Álvarez; Juan Carlos Erazo-Álvarez

parte de la organización tiempo - espacio por parte del infante y la sensibilidad para ser empático con sus semejantes.

\section{MÉTODO}

El diseño de la investigación responde a las dos modalidades, bibliográfico, documental, exploratorio con un enfoque mixto, y cohorte trasversal (Carreño-Godoy, Erazo-Álvarez, Narváez-Zurita, \& Moreno, 2020), siendo el universo como la muestra estadística 20 educadoras pertenecientes a los centros infantiles Dr. Cesar Molina del MIES y CEI Particular Universitario de Azogues, para identificar que estrategias aplican las educadoras para desarrollar la creatividad en los infantes de 12 a 36 meses, con métodos inductivo-deductivo, análisis documental (Pérez, Schenkel, \& Pérez, 2019).

En nivel exploratorio permitió examinar las técnicas grafoplásticas por medio de una revisión bibliográfica para determinar cuál es el grado de incidencia en el desarrollo de la creatividad de los niños, para ello se acudirá a las educadoras de los centros infantiles quienes podrán revelar cuál es el nivel de conocimiento acerca de las técnicas grafoplásticas, así como de su incidencia en el desarrollo de la creatividad de los niños.

Para la recolección de la información se aplicó la encuesta a las educadoras de los centros infantiles, por medio de un cuestionario creado con preguntas tipo escala de Likert, cuyo análisis e fiabilidad determinó un valor de 0.806 de Alfa de Cronbach (ErazoÁlvarez \& Narváez-Zurita, 2020).

Para la obtención y análisis de resultados se empleó el software SPSS y la utilización de pruebas estadísticas descriptivas (Vivar-Astudillo, Erazo-Álvarez, \& Narváez-Zurita, 2020). 
Revista Arbitrada Interdisciplinaria KOINONIA

Año 2020 . Vol V. $\mathrm{N}^{\circ} 1$. Especial Educación

Hecho el depósito de Ley: FA2016000010

ISSN: 2542-3088

FUNDACIÓN KOINONIA (F.K). Santa Ana de Coro. Venezuela.

Mirian-Del-Rocío González Espinoza; Darwin Gabriel García-Herrera; Cristián Andrés Erazo-Álvarez; Juan Carlos Erazo-Álvarez

\section{RESULTADOS}

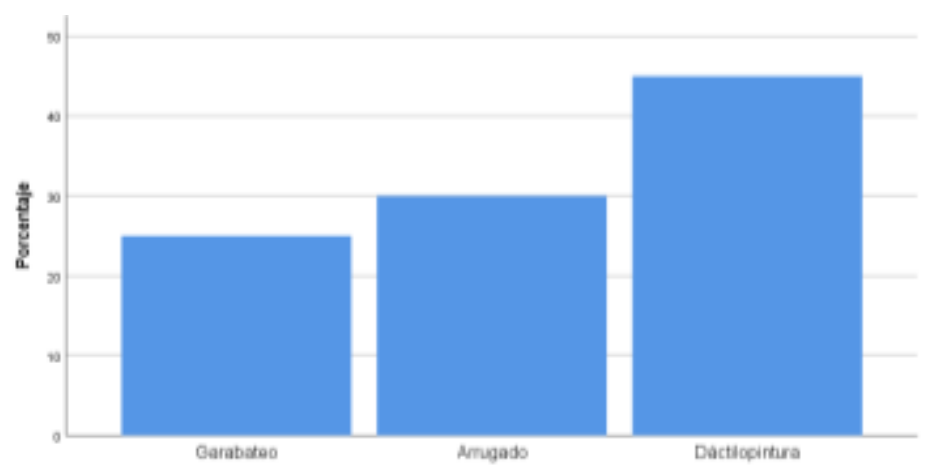

Figura 1. Técnicas grafoplásticas empleadas por las maestras. Fuente: Elaboración propia

De acuerdo con la figura 1, la técnica grafoplástica más utilizada por las educadoras para desarrollar la creatividad en los niños y niñas es la dactilopintura, en relación al arrugado y garabateo. Las docentes son conscientes que deben aplicar las técnicas grafoplásticas más innovadoras debido a que consideran que tienen una incidencia en el aprendizaje del infante, así como también del poder desarrollar la motricidad fina.

Nivel de significación

Se utiliza el nivel de 0,05 que representa una confiabilidad del 95\% Hipótesis planteadas:

(H1) Las técnicas grafo plásticas innovadoras están asociadas con el desarrollo artístico en niños y niñas de 12 a 36 meses, en los centros infantiles Dr. Cesar Molina y CEI Particular Universitario de Azogues.

(H0) Las técnicas grafo plásticas innovadoras no están asociadas al desarrollo artístico en los niños y niñas de 12 a 36 meses, en los centros infantiles Dr. Cesar Molina y CEI Particular Universitario de Azogues.

Para comprobar cuál de las hipótesis es verdadera se procede hacer pruebas de chicuadrado entre las preguntas escogidas que involucran a la variable tanto dependiente como independiente esto es: 
Revista Arbitrada Interdisciplinaria KOINONIA

Año 2020 . Vol V. $\mathrm{N}^{\circ} 1$. Especial Educación

Hecho el depósito de Ley: FA2016000010

ISSN: 2542-3088

FUNDACIÓN KOINONIA (F.K). Santa Ana de Coro. Venezuela.

Mirian-Del-Rocío González Espinoza; Darwin Gabriel García-Herrera; Cristián Andrés Erazo-Álvarez; Juan Carlos Erazo-Álvarez

\section{Tabla 1}

Relación entre estrategias para desarrollar la creatividad y cómo influye en el aprendizaje La creatividad influye en el aprendizaje de los niños

\begin{tabular}{|c|c|c|c|c|}
\hline & & Poco & Mucho & Total \\
\hline Estrategias & Libertad para que exprese & 3 & 0 & 3 \\
\hline desarrollar la creatividad & sus ideas & & & \\
\hline \multirow[t]{2}{*}{ en los niños } & Leer un cuento & 3 & 0 & 3 \\
\hline & Actividades artísticas & 1 & 13 & 14 \\
\hline Total & & 7 & 13 & 20 \\
\hline
\end{tabular}

Fuente: Elaboración propia.

De acuerdo a lo observado en la tabla 2, luego de hacer el respectivo análisis cruzado entre las variables; estrategias para desarrollar la creatividad y la creatividad influye en el aprendizaje de los niños, las educadoras manifiestan que aplican las estrategias que permiten incrementar el desarrollo creativo de los niños a través de la aplicación de las técnicas grafoplásticas innovadoras como son realizar: actividades artísticas, leer un cuento y, libertad para que expresen sus ideas; influyen favorablemente por cuanto tienen una incidencia en la creatividad influyendo en el proceso de aprendizaje de los niños aceptando la hipótesis; $\mathrm{HO}=$ Las técnicas grafoplásticas innovadoras no limitan el desarrollo artístico en los niños y niñas de 12 a 36 meses, es decir, que las técnicas grafoplásticas aplicadas desde los primeros años de vida del infante, es la base fundamental debido a que es el instante en que el niño comienza a experimentar y desarrollar destrezas y habilidades, así como su creatividad e imaginación.

\section{PROPUESTA}

Con base en los resultados donde se resalta el uso mayoritario de dactilopintura, es importante promover el uso de las técnicas grafoplásticas innovadoras que permitan incentivar la creatividad de los niños y niñas de 12 a 36 meses, aprovechando los desarrollos tecnológicos implícitos en la sociedad actual y permitan potencializar el desarrollo de la motricidad fina y por ende un desarrollo íntegro del niño. 


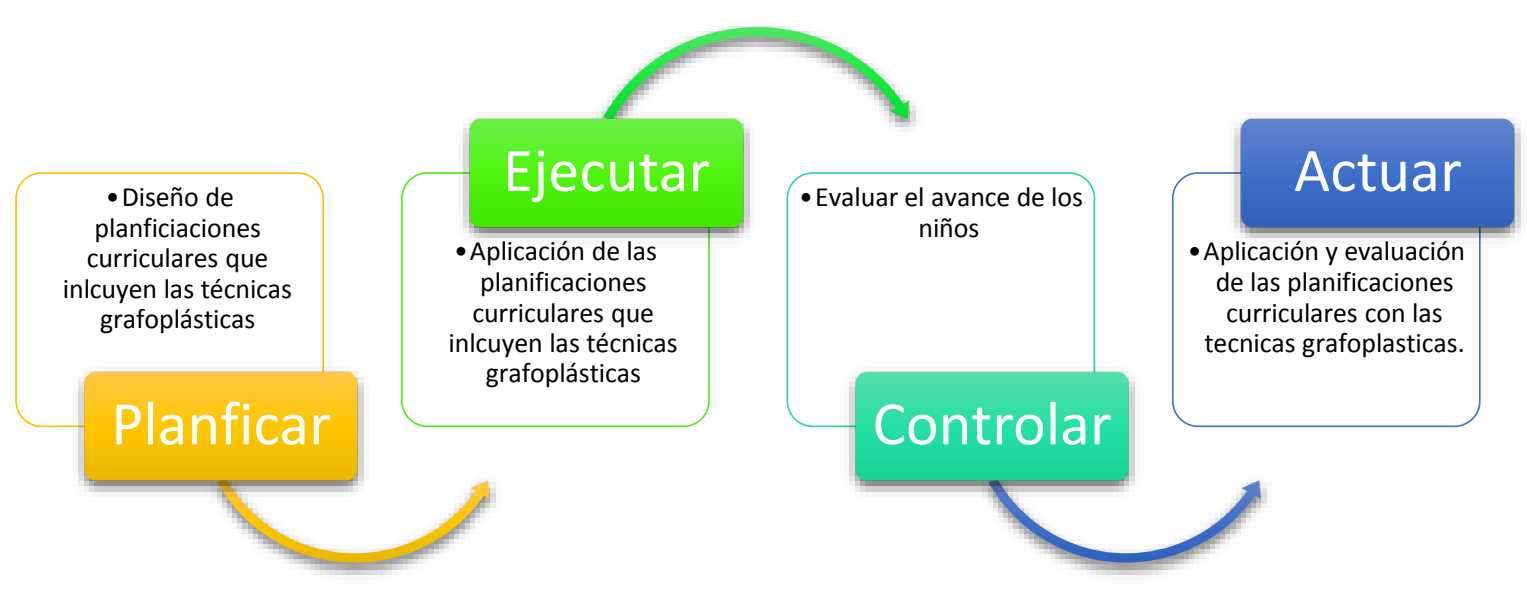

Figura 2. Aplicación de técnicas grafoplásticas innovadoras para incentivar la creatividad. Fuente: Elaboración propia

Planificar: Ofrecer a las autoridades educativas una alternativa viable que consiste en implementar dentro de la malla curricular las técnicas grafoplásticas innovadoras, que vayan encaminadas a desarrollar o potencializar las habilidades de la motricidad fina de los niños y niñas de 12 a 26 meses, al conocer que los infantes requieren realizar determinadas actividades donde la experimentación, entusiasmo y colaboración por descubrir e ir aprendiendo también puede ser divertido manteniendo toda la atención en las actividades que se lleven a cabo en las aulas de clase.

Así mismo, las guías didácticas y planificaciones de clase son los instrumentos que pueden emplear los docentes para trasmitir su pedagogía, pudiendo llegar a todos los niños el conocimiento, por ende, los infantes podrán ir desarrollando su crecimiento físico, mental y social en un ambiente agradable que sobretodo les permitan irse conociendo a sí mismos e ir utilizando su energía para desarrollar satisfactoriamente las actividades compartiendo con sus compañeritos y en general con todo su entorno.

Para ello se ha enfocado en realizar la propuesta con el fin de que los docentes puedan 
contar con varias alternativas estratégicas que buscan contribuir el desarrollo de la motricidad fina por medio de las técnicas grafoplásticas innovadoras.

Ejecutar: El desarrollar planificaciones educativas que incluyan predominantemente las técnicas grafoplásticas innovadoras como el uso de pintura y dibujo en dispositivos electrónicos como celulares, tablets o computadores, a través del aprovechamiento de softwares o apps diseñadas para este cometido como por ejemplo: Sunny Kid Games, Kids Doodle, Pintura para niños, Drawing Desk, WeDraw, entre otros; todo esto por medio de clases prácticas con el fin de que los beneficiarios directos sean los infantes, que comienzan a experimentar $\mathrm{e}$ ir desarrollando y potencializando las habilidades y creatividad que muchos las tienen escondidas, ahora bien, si se ejecutan las técnicas grafoplásticas innovadoras con el apoyo de tecnología los niños tendrán la posibilidad de experimentar nuevos escenarios que permiten incentivar la creatividad por medio del desarrollo de la motricidad fina, así como el mejoramiento de la aptitudes sociales y familiares, convirtiendo al niño y niña en seres felices, que puedan crecer aprendiendo de una forma divertida y amena acorde a la sociedad y entorno actual.

Controlar: El propósito fundamental de las técnicas grafoplásticas innovadores y su aplicación a través del uso de recursos tecnológicos fáciles y accesibles, es crear fácilmente diseños propios que nazcan de los niños y permitan expresar sus emociones. Para ello se puede recurrir a técnicas normales como dactilopintura y garabateo, mismas que se pueden ejecutar y controlar dentro de aplicaciones informáticas y permitan implícitamente el desarrollo de la creatividad y motricidad fina.

Actuar: A continuación, se procede a detallar las fases de implementación:

Diseño: Elaboración de las planificaciones educativas que contemplen el uso de técnicas grafoplásticas innovadoras y tecnológicas, de acuerdo a la Guía de orientaciones dadas en el currículo de educación inicial.

Tiempo de ejecución: Las planificaciones deben estar diseñadas una diferente para cada día de la semana, siguiendo una secuencia; y, repitiéndolas por las 4 semanas próximas. Evaluación: Luego de cada semana las educadoras emitirán una ficha de observación evaluando los avances que cada niño y niña en cuanto a su avance, desarrollo y 
Revista Arbitrada Interdisciplinaria KOINONIA

Año 2020. Vol V. ํ⒈ Especial Educación

Hecho el depósito de Ley: FA2016000010

ISSN: 2542-3088

FUNDACIÓN KOINONIA (F.K). Santa Ana de Coro. Venezuela.

Mirian-Del-Rocío González Espinoza; Darwin Gabriel García-Herrera; Cristián Andrés Erazo-Álvarez; Juan Carlos Erazo-Álvarez

expresión fácil de emociones en dibujos creativos.

\section{CONCLUSIONES}

Ahora bien, es importante conocer si los resultados obtenidos en la investigación que han reflejado la aceptación de la hipótesis que las técnicas grafoplásticas innovadoras tienen una incidencia en el desarrollo de la creatividad de los infantes que acuden a los centros infantiles que comparando resultados con los de la investigación realizada.

Las técnicas grafoplásticas innovadoras tienen una incidencia en el desarrollo de la creatividad del infante por cuanto en los primeros años de vida son en los que se debe tener mayor énfasis en un buen crecimiento y que por medio de las técnicas grafoplásticas innovadoras se puede lograr potencializar aspectos de desarrollo de la motricidad fina, habilidades y destrezas mentales y psicosociales asegurando un mejor desempeño del infante.

Las técnicas grafoplásticas innovadoras juegan un papel muy importante durante el proceso biológico de crecimiento de los infantes, cada fase de crecimiento debe ser considerada en el instante de aplicar adecuadamente las técnicas grafoplásticas innovadoras a pesar de los varios beneficios que aportan en la ciudad de Azogues todavía no forman parte en las mallas curriculares debido a varios factores como el no contar con las facilidades a los materiales o incluso a la no auto capacitación y poco interés del personal docente que labora en los centros infantiles.

Cuando aplican las actividades que fomentan el desarrollo de la creatividad tienen una incidencia positiva para los infantes teniendo en cuenta que no solo se desarrolla la motricidad fina sino también aspectos como habilidades mentales, sociales y familiares.

Se hace necesario realizar acciones para que las técnicas grafoplásticas innovadoras sean incluidas en las aulas de clase lo que requiere también que las educadoras responsables posean la capacidad y conocimiento para aplicar correctamente las técnicas grafoplásticas que les permitan seguir desarrollando y porque no decir impulsar las habilidades innatas de los infantes. 


\section{FINANCIAMIENTO}

No monetario.

\section{AGRADECIMIENTO}

A la Universidad Católica de Cuenca, por impulsar del desarrollo de la investigación.

\section{REFERENCIAS CONSULTADAS}

Asamblea Nacional Constituyente. (2012). Reglamento a la Ley Orgánica de Educación Intercultural [Regulation to the Organic Law of Intercultural Education]. Quito: Registro Oficial. Obtenido de https://n9.cl/bca5

Cabrera, V., \& Garcia, D. (2019). El desarrollo de la motricidad fina en los niños y niñas del grado preescolar [The development of fine motor skills in preschool children]. Mendive revista de educacion, 225-226. Obtenido de https://n9.cl/q4et

Carreño-Godoy, M. J., Erazo-Álvarez, J. C., Narváez-Zurita, C. I., \& Moreno, V. P. (2020). La responsabilidad social en las empresas camaroneras [Social responsibility in shrimp companies]. Revista Arbitrada Interdisciplinaria Koinonía, 455-482. doi:http://dx.doi.org/10.35381/r.k.v5i10.702

Castañeda, M. (2013). Desarrollo artístico [Artistic development]. Bogota: Fred.

Chuva Castillo, P. G. (2016). Desarrollo de la motricidad fina a través de las técnicas grafoplásticas [Fine motor development through graphoplastic techniques]. Cuenca: Universidad Politécnica Salesiana. Obtenido de https://dspace.ups.edu.ec: https://n9.cl/mt1u

Educiudadania. (2016). Plan Decenal de Educación [Ten-Year Education Plan]. Obtenido de https://n9.cl/fbvx

Erazo-Álvarez, J. C., \& Narváez-Zurita, C. I. (2020). Medición y gestión del capital intelectual en la industria del cuero - calzado en Ecuador. [Measurement and management of intellectual capital in the leather industry - footwear in Ecuador]. Revista Arbitrada Interdisciplinaria Koinonía, 437-467. doi:http://dx.doi.org/10.35381/r.k.v5i9.662

Gamarra, P., \& Navas, L. (2017). La detección temprana de niños superdotados del nivel de Educación Inicial en el contexto peruano [Early detection of gifted children at the Initial Education level in the Peruvian context]. RUA. Obtenido de https://n9.cl/4pfb9 
Gancino, R. (2012). Grafoplastia [Graphoplasty]. Quito: Lasplast.

Gil Madrona, P. (2008). Habilidades en la infancia y su desarrollo desde una educación fisica animada [Skills in childhood and their development from an animated physical education]. Revista Iberomaerica de Educación, 1-26. Obtenido de https://n9.cl/71jc

Hussey, W. (2017). Pensamiento creativo: Actividades estimulantes, i increíbles!, para niños de 6 a 12 años [Creative Thinking: Stimulating, Incredible! Activities for Kids Ages 6-12]. Narcea. Obtenido de https://n9.cl/yxd2

Larrea, P. (2013). Educación Artística [Artistic education]. Cuenca: Navas.

Ministerio de Educación del Ecuador. (2019). Curriculo de Educación Inicial [Initial Education Curriculum]. Obtenido de https://educacion.gob.ec/educacion-inicial/

Murrillo, G. (2012). Técnicas Grafoplásticas [Graphoplastic techniques]. Riobamba: Polinesa.

Pérez, E., Schenkel, E., \& Pérez, M. I. (2019). Un abordaje teórico de la investigación cualitativa como enfoque metodológico [A theoretical approach to qualitative research as a methodological approach]. Acta Geográfica, 227-233. doi:http://dx.doi.org/10.5654/acta.v12i30.5201

Physical Development and Health. (2017). Teaching Strategies That Support Fine-Motor Skills [Estrategias de enseñanza que apoyan las habilidades motoras finas]. Obtenido de https://n9.cl/y7drn

Subsecretaría de Educación Inclusiva. (2019). Educación Inclusiva [Inclusive education]. Obtenido de https://n9.cl/772e8

Vivar-Astudillo, A. Y., Erazo-Álvarez, J. C., \& Narváez-Zurita, C. I. (2020). La cadena de valor como herramienta generadora de ventajas competitivas para la Industria Acuícola [The value chain as a tool generating competitive advantages for the Aquaculture Industry]. Revista Arbitrada Interdisciplinaria Koinonía, 4-33. doi:http://dx.doi.org/10.35381/r.k.v6i10.686 